\title{
STUDY OF SUN'S 'HYDROMAGNETIC' OSCILLATIONS USING SUNSPOT DATA
}

\author{
M.H.GOKHALE, J.JAVARAIAH and K.M.HIREMATH \\ Indian Institute of Astrophysics \\ Bangalore 560034 \\ India
}

\begin{abstract}
Spherical-harmonic-fourier (SHF) analysis of sun's 'nominal toroidal magnetic field', computed using sunspot data during 1874-1976, shows that sunspot activity can be considered as possibly originating in interference of sun's axi-symmetric 'hydromagnetic' oscillations of odd degrees up to $\ell \approx 21$ and periods $\approx 22 y$. The relative amplitudes and relative phases of these oscillations remain fairly constant, leading to butterfly diagrams with stable latitude-time correlations on all latitude scales $29^{\circ}$. The amplitudes and phases do however undergo slow coherent variations. The main modes ( $\ell 111$ ) represent an approximately standing oscillation.

The mean power spectrum can be fitted excellently to the form a $\ell^{3} \exp (-b \ell)$ expected for waves trapped between the poles.
\end{abstract}

\section{Introduction}

In an earlier paper (Gokhale and Javaraiah, 1989) we used sunspot data during 1902-1954 to show that SHF analysis of 'sunspot occurrence probability' yields approximately same relative amplitudes and relative phases, during different sunspot cycles, for axisymmetric modes of '11y' periodicity with even degrees up to $\ell \approx 22$, in which most of the SHF power is concentrated. This was shown to be a consequence of a similar stability of relative amplitudes and relative phases of axisymmetric modes, of ' 22 $y$ ' period and odd degrees at least up to $\ell \approx 11$, in a 'nominal toroidal magnetic field' defined by attaching to the sunspot occurrence probability signs $(t /-)$ according to Hale's laws of magnetic polarities. Reasons were given to believe that these modes represent slow MHD oscillations of the sun.

In this paper we present results of the SHF analysis of the 'nominal toroidal field' for the 82 intervals of $22 \mathrm{y}$ length during 1874-1976, each interval being displaced by 
one year with respect to the previous one. We also discuss physical meaning of the modes and of the energy spectrum.

\section{Data and Method of Analysis}

The heliographic colatitudes $\theta_{i}$,longitudes $\phi_{i}$, and epochs $t_{i}$ (in days and fractions from 0.0 UT of January 1, 1874), for each sunspot group, on each day of its observation,were determined using relevant information from a magnetic tape of the Greenwich photo-heliographic data which was kindly provided to us by H.Balthassar.

Sunspot occurrence probability ' $p$ ' $(\theta, \phi, t)$ during any interval ( $T_{1}, T_{2}$ ) is defined (paper $I$ ) as described by a delta function at each data point $\left(\mu i, \phi i, \tau_{i}\right)$ in $(\mu, \phi, \tau)$ space, multiplied by the reciprocal of the total number of data points during $\left(T_{1}, T_{2}\right)$, where $\mu=\cos \theta$ and $\tau=t_{i} /\left(T_{2}-T_{1}\right)$.

The 'nominal toroidal field' $\mathrm{B} \varphi(\theta, \phi, t)$ was determined from $p(\theta, \phi, t)$ as in paper I(see sec.1 above).The SHF coefficients of $\mathrm{B} \varphi(\theta, \phi, t)$ are also computed using the orthonormality of the SHF functions as described in paper $I$.

\section{Results and Discussion}

As in paper $I$, most of the SHF power is found to be concentrated in axisymmetric modes of odd degrees with ' $22 y$ ' periodicity.

The amplitudes of these modes during the 82 intervals of $22 y$ each, (1874-1895, 1875-1896, etc.) remain in the ranges shown in Fig 1 (a). The relative phases of these modes during the 82 intervals are shown in Fig. $1(\mathrm{~b})$.

These results confirm the conclusion in paper I that $p(\theta, \phi, t)$ is related to $B \varphi(\theta, \phi, t)$ in a nonlinear way as $\left.\mathrm{p}(\theta, \phi, t) \approx \mathrm{CB}_{\varphi}^{2}(\theta, \phi, t)\right]^{1 / 2}$ where $\mathrm{B} \varphi(\theta, \phi, t)$ can be considered as given by superposition of ' 22 y' periodic axisymmetric modes of odd degrees at least up to $l \approx 21$ whose relative amplitudes and relative phases remain approximately constant so as to yield stable butterfly diagrams in p $(\theta, \phi, t)$. The main modes up to $\ell \approx 9$ describe an approximately stationary oscillation, since their relative phases are near $0^{\circ}$ or $180^{\circ}$.

In order to illustrate the significance of the constancy of the relative amplitudes and phases we compare these with those in Figures 2 (a), $2(b)$ as computed from a simulated data set in which the values of $t_{i}$ are retained as in the real data but the values of the latitudes $\lambda_{i}\left(=90^{\circ}-\theta_{i}\right)$ are simulated as follows: $\lambda_{i}=$ (random sign) $x\left[\lambda_{0}+\Delta \lambda_{i}\right]$, in which, during the first seven years of each sunspot cycle, io reduces linearly from $25^{\circ}$ to $10^{\circ}$ and $\Delta \lambda_{i}$ are random values between $\left(-9^{\circ},+9^{\circ}\right)$. During the last four years of each cycle, $\lambda_{0}$ remalns constant at $8^{\circ}$ and $\Delta \lambda_{i}$ has random values 
between $\left(-6^{\circ},+6^{\circ}\right)$.

From comparison of Figures 1 and 2 it is clear that the constancy and the values of the amplitudes and phases of the modes up to $\& \approx 13$ can be reproduced by the simulated data. This is because the systematic variation of $\lambda_{0}$ truly reproduces the latitude-time correlation in the butterfly diagram on sciales $\geq 10^{\circ}$. However in Fig.2 the power in the modes $\ell, 13$ is much smaller than that in Fig.1. Also their phase variations become increasingly irregular, till at

(a)

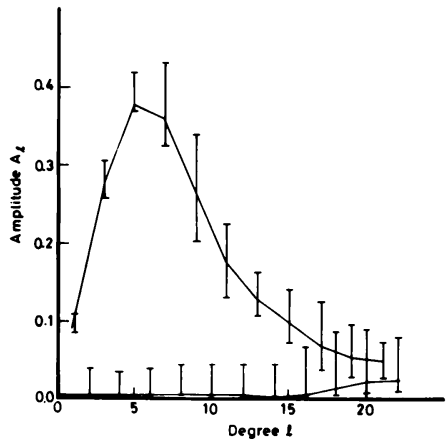

(b)

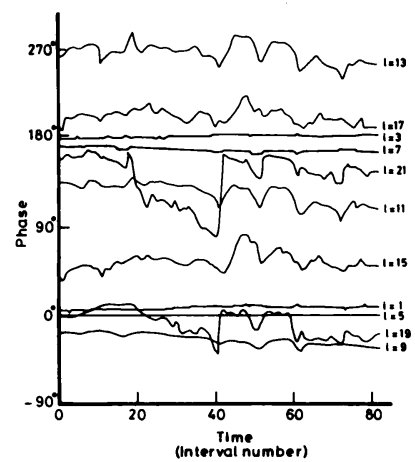

Figure 1. (a) Spectrum of relative amplitudes of the axisymmetric modes of $22 y$ periodicity in ' $B \psi$ ' The bars represent total scatter of the 82 values corresponding to the 82 intervals. The continuous curve represents the values obtained from the whole data set of the 103 years. (b) Relative phases $\left(\varphi \frac{i}{i}\right)$.

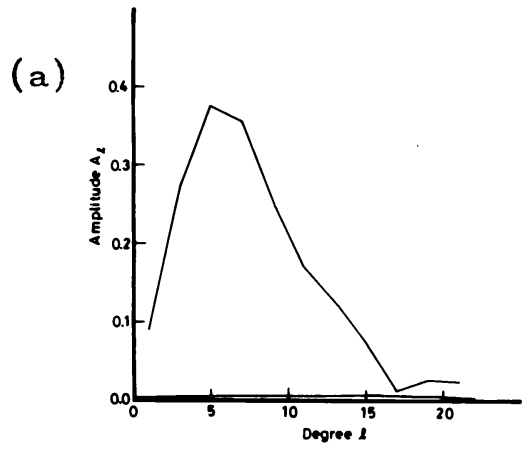

Figure 2. (a) Relative amplitudes and (b) relative phases obtained from the 'simulated' data. (b)

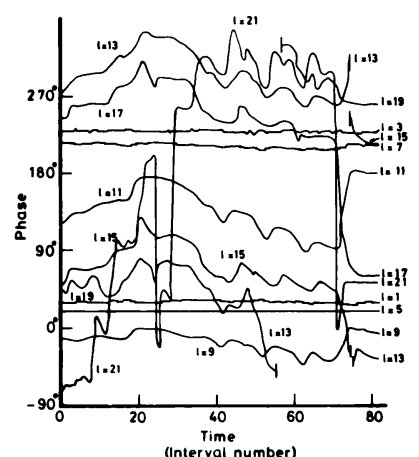


l=21, it becomes extremely irregular. This shows that the value of the amplitudes and the constancy of phases upto $\ell \approx 21$ cannot be obtained by a simulated data set unless it reproduces $(\theta-t)$ correlations on all scales down to $\sim 9^{\circ}$.

Such a simulation will need ad-hoc 'systematic'assumptions just for reproducing those correlations. It is much simpler and straightforward to conclude that the correlations in the real $\theta_{i}$ and $t i$ result from interference of the global oscillations of $\mathrm{B} \varphi$ with amplitudes and phases as given by the SHF analysis. (We use the word "interference" since $p(\theta, \phi, t)$ is non-linearly related to $\mathrm{B} \varphi(\theta, \phi, t))$.

\section{Physical significance}

\subsection{PHYSICAL NATURE OF THE OSCILlations}

As pointed out in paper $I$, the period indicates slow-MHD nature of the modes which requires these to be torsional if the basic field is poloidal. However these oscillations may have both toroidal and meridional velocity components, constituting essentially an "MHD dynamo".

\section{2 'REALITY' OF THE 'NOMINAL' TOROIDAL FIELD}

The 'nominal toroidal field' need not represent the real toroidal field. Yet it seems that it does so, since its spatial and temporal frequencies, as well as the amplitude spectrum, are similar to those of the radial field derived from full disc magnetograms (Stenflo, 1988).

\subsection{THE FORM OF THE ENERGY SPECTRUM}

The slow MHD waves responsible for these oscillations must be trapped between the poles, with small phase shifts and dissipation. The trapping will lead to a power spectrum $\sim a \ell^{2}$ in the small $\ell$ domain and possibly $\sim a \ell^{3} \exp (-b \ell)$ in the large $\ell$ domain. The power spectrum given by squares of the amplitudes in Fig.1 can be fitted excellently < $\chi^{2}$-confidence $>99.98 \%)$ to the form $a \ell^{3} \exp (-b \ell)$ with $a=0.0142$ and $b=0.552 \pm 0.015$.

\section{References}

Gokhale, M.H. and Javaraiah, J (1989) 'Sunspot activity as originating in interference of sun's global MHD oscillations', paper submitted to MNRAS.

Stenflo,J.O. (1988) 'Global wave patterns in the sun's magnetic field', Astrophys \& Sp. Sci., 144, 321. 